\title{
Discrete Tomography: Magic Numbers for N-Fold Symmetry
}

\author{
C. Huck, M. Moll And J. Nilsson \\ Fakultät für Mathematik, Universität Bielefeld, Postfach 100131, 33501 Bielefeld, Germany
}

\begin{abstract}
We consider the problem of distinguishing convex subsets of $n$-cyclotomic model sets $\Lambda$ by (discrete parallel) $\mathrm{X}$-rays in prescribed $\Lambda$-directions. In this context, a 'magic number' $m_{\Lambda}$ has the property that any two convex subsets of $\Lambda$ can be distinguished by their X-rays in any set of $m_{\Lambda}$ prescribed $\Lambda$-directions. Recent calculations suggest that (with one exception in the case $n=4$ ) the least possible magic number for $n$-cyclotomic model sets might just be $N+1$, where $N=\operatorname{lcm}(n, 2)$.
\end{abstract}

DOI: $10.12693 /$ APhysPolA.126.486

PACS: 61.43.-j, 61.44.Br

\section{Introduction}

Discrete tomography (DT) is concerned with the inverse problem of retrieving information about some finite set in Euclidean space from (generally noisy) information about its slices. One important problem is the unique reconstruction of a finite point set in Euclidean 3-space from its (discrete parallel) $X$-rays in a small number of directions, where the $X$-ray of the finite set in a certain direction is the line sum function giving the number of points in the set on each line parallel to this direction.

The interest in the discrete tomography of planar Delone sets $\Lambda$ with long-range order is motivated by the requirement in materials science for the unique reconstruction of solid state materials like quasicrystals slice by slice from their images under quantitative high resolution transmission electron microscopy (HRTEM). In fact, a technique is described in [1, 2], which can, for certain crystals, effectively measure the number of atoms lying on densely occupied columns. Since we aim at a slice by slice approach, it is sufficient to study the DT of planar Delone sets with long-range order.

Since different finite subsets of $\Lambda$ may have the same $\mathrm{X}$-rays in several directions, one is naturally interested in conditions to be imposed on the set of directions together with restrictions on the possible finite subsets of $\Lambda$ such that the latter phenomenon cannot occur. Here, we consider the convex subsets of so-called strong cyclotomic Delone sets $\Lambda$ and present new results on the problem of distinguishing convex subsets of $\Lambda$ by X-rays in prescribed $\Lambda$-directions. It is well known [3] that there are four prescribed $\Lambda$-directions such that any two convex subsets of $\Lambda$ can be distinguished by the corresponding $\mathrm{X}$-rays, whereas less than four $\Lambda$-directions never suffice for this purpose. Moreover, there is a finite number $m_{\Lambda}$ such that any two convex subsets of $\Lambda$ can be distinguished by their X-rays in any set of $m_{\Lambda}$ prescribed $\Lambda$ directions. It was essentially shown in [3] that the least possible 'magic numbers' $m_{\Lambda}$ in the case of the practically most relevant examples of strong $n$-cyclotomic Delone sets $\Lambda$ with $n=5,8$ and 12 only depend on $n$ and are (in that order) 11, 9 and 13; see also [4] for a gentle introduction. This extended a well-known result of
Gardner and Gritzmann [5] on the corresponding problem for the crystallographic cases $n=3,4$ (with least possible number $m_{\Lambda}=7$ in both cases) to cases that are relevant in quasicrystallography. With the exception of the case $n=4$, the explicit calculations above indicate a very simple relation between $n$ and the associated least possible 'magic numbers', i.e. for the cases $n=3,5,8$ and 12 the magic number is just $N+1$, where $N=\operatorname{lcm}(n, 2)$. In this short note, we present further computational evidence that this might be true in general. For detailed proofs and an extensive list of references, we refer the reader to [3].

\section{Cyclotomic Delone sets}

Throughout, the Euclidean plane is identified with the complex numbers. We denote by $K_{\Lambda}$ the smallest subfield of $\mathbb{C}$ that contains the rational numbers as well as the union of $\Lambda-\Lambda$ and its image $\overline{\Lambda-\Lambda}$ under complex conjugation. For $n \in \mathbb{N}$, we always let $\zeta_{n}=\mathrm{e}^{2 \pi \mathrm{i} / n}$, a primitive $n$-th root of unity in $\mathbb{C}$. Then, the smallest subfield of $\mathbb{C}$ that contains the rational numbers as well as $\zeta_{n}$ is the $n$-th cyclotomic field denoted by $\mathbb{Q}\left(\zeta_{n}\right)$. The latter is just the $\mathbb{Q}$-span of the $n$-th roots of unity and thus contains the $\mathbb{Z}$-span $\mathbb{Z}\left[\zeta_{n}\right]$ of the $n$-th roots of unity. Note that $\mathbb{Q}\left(\zeta_{n}\right)=\mathbb{Q}\left(\zeta_{N}\right)$, where $N=\operatorname{lcm}(n, 2)=[n, 2]$ is the least common multiple of $n$ and 2. Recall that a homothety of the complex plane is given by $z \mapsto \lambda z+t$, where $\lambda \in \mathbb{R}$ is positive and $t \in \mathbb{C}$. For our purposes, the following rather abstract definition is convenient.

Definition 1. Let $n \geq 3$. A Delone set $\Lambda \subset \mathbb{C}$ is called a strong $n$-cyclotomic Delone set if it satisfies the following properties:

$\left(n-C y c^{*}\right) K_{\Lambda}=\mathbb{Q}\left(\zeta_{n}\right)$.

(Hom) For any finite subset $F$ of $K_{\Lambda}$, there is a homothety $h$ of the complex plane that maps the elements of $F$ to $\Lambda$.

Further, $\Lambda$ is called a strong cyclotomic Delone set if it is a strong $n$-cyclotomic Delone set for a suitable $n \geq 3$.

This includes many of the commonly used mathematical models of slices that occur in quasicrystallography, i.e. the $n$-cyclotomic model sets $\Lambda \subset \mathbb{Z}\left[\zeta_{n}\right]$; cf. $[3,6]$. For 
suitable choices of the window, these sets have $N$-fold rotational symmetry. They range from periodic examples like the fourfold square lattice $(n=4)$ or the sixfold triangular lattice $(n=3)$ to nonperiodic examples like the vertex set of the tenfold Tübingen triangle tiling $(n=5)$, the eightfold Ammann-Beenker tiling $(n=8)$ or the twelvefold shield tiling $(n=12)$; see Fig. 1 . The vertex sets of Penrose tilings fail to be cyclotomic model sets but are still strong 5-cyclotomic Delone sets [7].

\section{Discrete tomography}

The unit circle in $\mathbb{C}$ is denoted by $\mathbb{S}^{1}$ and its elements are also called directions.

Definition 2. Let $F$ be a finite subset of $\mathbb{C}$, let $u \in \mathbb{S}^{1}$ be a direction, and let $\mathcal{L}_{u}$ be the set of lines in the complex plane in direction $u$. Then the (discrete parallel) X-ray of $F$ in direction $u$ is the function $X_{u} F: \mathcal{L}_{u} \rightarrow \mathbb{N}_{0}=$ $\mathbb{N} \cup\{0\}$, defined by

$$
X_{u} F(\ell)=\operatorname{card}(F \cap \ell) .
$$

Furthermore, we say that the elements of a collection $\mathcal{F}$ of finite subsets of $\mathbb{C}$ are determined by the $X$-rays in the directions of a finite set $U \subset \mathbb{S}^{1}$ of directions if different elements of $\mathcal{F}$ cannot have the same $X$-rays in the directions of $U$.

Let $\Lambda \subset \mathbb{C}$ be a fixed Delone set. Obviously, only $\Lambda$ directions (directions parallel to nonzero elements of the difference set $\Lambda-\Lambda$ ) are reasonable. One can see that, for a strong $n$-cyclotomic Delone set $\Lambda$, the set of $\Lambda$ directions coincides with the set of $\mathbb{Q}\left(\zeta_{n}\right)$-directions (defined analogously). Note that the present text is only concerned with the uniqueness problem of determining the elements of a large collection of finite subsets of $\Lambda$ by few X-rays in prescribed $\Lambda$-directions. For the algorithmic reconstruction problem in the quasicrystallographic setting, see [8].

One can easily see that no finite set of pairwise nonparallel $\Lambda$-directions suffices in order to determine the whole class of finite subsets of a (strong) cyclotomic Delone set $\Lambda$ (in contrast to strong cyclotomic Delone sets, cyclotomic Delone sets only satisfy the weaker condition $\left.K_{\Lambda} \subset \mathbb{Q}\left(\zeta_{n}\right)\right)$ by the corresponding X-rays. It has proven to be most fruitful to focus on the convex subsets of (strong) cyclotomic Delone sets. The latter are bounded (and thus finite) subsets $C$ of $\Lambda$ with $C=\operatorname{conv}(C) \cap \Lambda$, where $\operatorname{conv}(C)$ denotes the convex hull of $C$.

\section{Determining convex sets by X-rays}

Definition 3. For a finite set $U \subset \mathbb{S}^{1}$ of directions, a nondegenerate convex polygon $P \subset \mathbb{C}$ is called a $U$ polygon if it has the property that whenever $v$ is a vertex of $P$ and $u \in U$, the line in the complex plane in direction $u$ which passes through $v$ also meets another vertex $v^{\prime}$ of $P$. For a subset $S \subset \mathbb{C}, P$ is called a $U$-polygon in $S$, if its vertices lie in $S$.
One has the following fundamental result.

Theorem 1. [3], Fact 5.3. Let $\Lambda$ be a (strong) cyclotomic Delone set and let $U \subset \mathbb{S}^{1}$ be a set of two or more pairwise nonparallel 1 -directions. The following statements are equivalent:

(i) The convex subsets of $\Lambda$ are determined by the $X$ rays in the directions of $U$.

(ii) There is no U-polygon in $\Lambda$.

If, in addition, $\operatorname{card}(U)<4$, there is a $U$-polygon in $\Lambda$.

Note that the proof of direction (ii) $\Rightarrow$ (i) needs property (Hom); see Fig. 1 for an illustration of the other (easy) direction (i) $\Rightarrow($ ii). Thus, one is immediately led to the investigation of $U$-polygons.

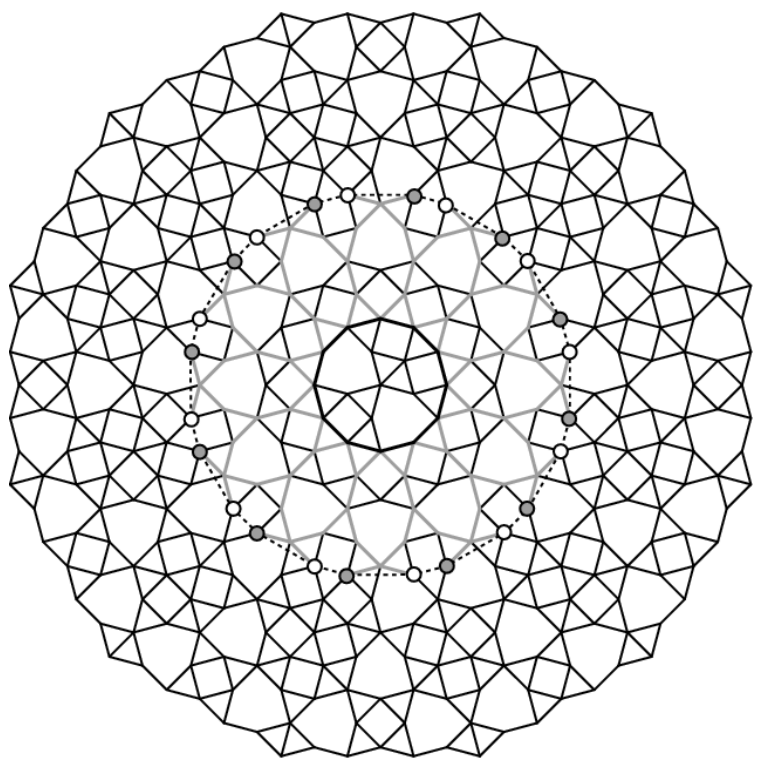

Fig. 1. The construction of a $U$-polygon in the vertex set $\Lambda$ of the twelvefold shield tiling (as described in the paragraph preceding Proposition 1), where $U$ is the set of $N=12 \Lambda$-directions given by the edges and diagonals of the central regular dodecagon. Here, the points obtained by translating the regular $N$-gon are already vertices of the tiling and not only elements of $\mathbb{Q}\left(\zeta_{n}\right)$. The vertices of $\Lambda$ in the interior of the $U$-polygon together with the vertices indicated by the white and grey dots, respectively, give two different convex subsets of $\Lambda$ with the same X-rays in the directions of $U$.

\section{Existence of $U$-polygons}

For $n \geq 3$, consider the regular $N$-gon $R$ inscribed in the unit circle, with one vertex at 1 . Now attach $N$ translates of $R$ edge-to-edge to $R$ in the obvious way. Then, one can easily verify that the convex hull $P$ of the resulting point set is a $U$-polygon in $\mathbb{Q}\left(\zeta_{n}\right)$, where $U$ is the set of $N$ pairwise nonparallel $\mathbb{Q}\left(\zeta_{n}\right)$-directions given by the edges and diagonals of $R$. Now let $\Lambda$ be a strong 
$n$-cyclotomic Delone set. Employing properties $\left(n-\mathrm{Cyc}^{*}\right)$ and (Hom), one obtains a $U$-polygon in $\Lambda$ for a set $U$ consisting of $N \Lambda$-directions; compare Fig. 1 . In view of Theorem 1, this proves that the magic number for strong $n$-cyclotomic Delone sets is at least $N+1$.

Proposition 1. Let $\Lambda$ be a strong $n$-cyclotomic Delone set. Then there is a $U$-polygon in $\Lambda$ with $\operatorname{card}(U)=N$.

Definition 4. Let $\left(t_{1}, t_{2}, t_{3}, t_{4}\right)$ be an ordered tuple of four distinct elements of $\mathbb{R} \cup\{\infty\}$. Then, its cross ratio $\left\langle t_{1}, t_{2}, t_{3}, t_{4}\right\rangle$ is

$$
\left\langle t_{1}, t_{2}, t_{3}, t_{4}\right\rangle=\frac{\left(t_{3}-t_{1}\right)\left(t_{4}-t_{2}\right)}{\left(t_{3}-t_{2}\right)\left(t_{4}-t_{1}\right)} \in \mathbb{R}^{*},
$$

with the usual conventions if one of the $t_{i}$ equals $\infty$.

By construction, the cross ratio of slopes of four pairwise nonparallel $\Lambda$-directions is an element of the field $K_{\Lambda} \cap \mathbb{R}$. In case of (strong) $n$-cyclotomic Delone sets $\Lambda$, these cross ratios are thus elements of the field $\mathbb{Q}\left(\zeta_{n}\right) \cap \mathbb{R}$.

Employing a blend of methods from the theory of cyclotomic fields and previous results obtained by Gardner and Gritzmann [5], one obtains the following deep result on $U$-polygons. The novelty here is the assertion on the formula for the least possible upper bounds $b_{n}$ and the largest possible sets $U$ of directions. These were found by direct computation (the proof of [3], Thm. 5.6, immediately leads to an algorithm).

Theorem 2. [3], Thm. 5.7. Let $n \geq 3$ and let $\Lambda$ be a strong n-cyclotomic Delone set. Further, let $U \subset \mathbb{S}^{1}$ be a set of four or more pairwise nonparallel $\Lambda$-directions and suppose the existence of a U-polygon. Then, the cross ratio of slopes of any four directions of $U$, arranged in order of increasing angle with the positive real axis, is an element of the finite set $\mathcal{C}_{[2 n, 12]}\left(\mathbb{Q}\left(\zeta_{n}\right) \cap \mathbb{R}\right)$ of numbers in the field $\mathbb{Q}\left(\zeta_{n}\right) \cap \mathbb{R}$ that can be written in the form

$$
\frac{\left(1-\zeta_{[2 n, 12]}^{k_{1}}\right)\left(1-\zeta_{[2 n, 12]}^{k_{2}}\right)}{\left(1-\zeta_{[2 n, 12]}^{k_{3}}\right)\left(1-\zeta_{[2 n, 12]}^{k_{4}}\right)}
$$

where $\left(k_{1}, k_{2}, k_{3}, k_{4}\right) \in \mathbb{N}^{4}$ satisfies

$$
k_{3}<k_{1} \leq k_{2}<k_{4} \leq[2 n, 12]-1
$$

and

$$
k_{1}+k_{2}=k_{3}+k_{4} .
$$

Moreover, $\operatorname{card}(U)$ is bounded above by a finite number $b_{n} \in \mathbb{N}$ that only depends on $n$. With the exception of $b_{4}=7$, for $3 \leq n \leq 50$ and $n=61, b_{n}=N=[n, 2]$ is best possible. Moreover, for these values of $n$, if there is a $U$-polygon with $\operatorname{card}(U)=N$, then there is a linear automorphism of the complex plane that takes the directions from $U$ to a set of vectors which when normalised are given by $\mathrm{e}^{h \pi \mathrm{i} / N}$, where $h \in\{0,1,2, \ldots, N-1\}$ (compare Fig. 1).

\section{Result}

Theorems 1 and 2 now immediately imply our main result on the determination of convex subsets of strong cyclotomic Delone sets.

Theorem 3. [3], Thm. 5.11. Let $n \geq 3$ and let $\Lambda$ be $a$ strong n-cyclotomic Delone set.

(a) There are sets of four pairwise nonparallel $\Lambda$ directions such that the convex subsets of $\Lambda$ are determined by the corresponding $X$-rays. In addition, less than four pairwise nonparallel $\Lambda$-directions never suffice for this purpose.

(b) There is a finite number $m_{n} \in \mathbb{N}$ that only depends on $n$ such that the convex subsets of $\Lambda$ are determined by the $X$-rays in any set of $m_{n}$ pairwise nonparallel $\Lambda$-directions. With the exception of $m_{4}=7$, for $3 \leq n \leq 50$ and $n=61, m_{n}=N+1$ is best possible.

\section{Recipe and conjecture}

By Theorems 1 and 2 above, it suffices for Theorem 3(a) to take any set of four pairwise nonparallel A-directions such that the cross ratio of their slopes, arranged in order of increasing angle with the positive real axis, is not an element of the finite set $\mathcal{C}_{[2 n, 12]}\left(\mathbb{Q}\left(\zeta_{n}\right) \cap \mathbb{R}\right)$ which can be computed directly. E.g. for the vertex set of the shield tiling (a 12-cyclotomic model set) below, the set $\mathcal{C}_{24}(\mathbb{Q}(\sqrt{3}))$ of cross ratios to be avoided is given by (note that the number $\frac{2}{\sqrt{3}}$ is mistakenly missing in the corresponding list appearing in [3] Cor. 4.10(c))

$$
\begin{aligned}
& \left\{8-4 \sqrt{3}, \frac{3+2 \sqrt{3}}{6}, \frac{-3+3 \sqrt{3}}{2}, \frac{2}{\sqrt{3}}, \frac{3+\sqrt{3}}{4},\right. \\
& \frac{2+\sqrt{3}}{3}, 3-\sqrt{3}, \frac{4}{3}, \frac{1+\sqrt{3}}{2},-2+2 \sqrt{3}, \frac{3}{2}, \frac{3+\sqrt{3}}{3}, \\
& \sqrt{3}, \frac{2+\sqrt{3}}{2}, 2, \frac{3+2 \sqrt{3}}{3}, \frac{3+\sqrt{3}}{2}, 1+\sqrt{3}, 3, \\
& \frac{6+2 \sqrt{3}}{3}, 2+\sqrt{3}, 4,3+\sqrt{3}, \frac{5+3 \sqrt{3}}{2}, 3+2 \sqrt{3}, \\
& 4+2 \sqrt{3}, 6+3 \sqrt{3}, 7+4 \sqrt{3}, 8+4 \sqrt{3}\} .
\end{aligned}
$$

See [3], Cor. 4.10, for concrete results in the other practically important cases $n=5,8$ of quasicrystallography.

Conjecture 1. With the exception of $m_{4}=7$, the trivial lower bound $m_{n}=N+1$ is the magic number for strong $n$-cyclotomic Delone sets for all $n \geq 3$, where $N=[n, 2]$.

The above exception can be explained as follows. For the two crystallographic cases $n=3,4$, the corresponding $n$-cyclotomic model sets, i.e. translates of the triangular $(N=6)$ resp. square lattice $(N=4)$, are affinely equivalent. It is thus clear that, as it is the case for the triangular lattice, also the square lattice contains a $U$-polygon for a set $U$ consisting of $\operatorname{six}$ (and not only $N=4$ ) lattice directions; cf. [5]. 


\section{Seven a universal magic number in $3 \mathrm{D}$ ?}

It is certainly of interest to abandon the slice-by-slice approach to the problem of uniquely reconstructing convex subsets of $3 \mathrm{D}$ Delone sets by X-rays and to turn to sets $U \subset \mathbb{S}^{2}$ of $\Lambda$-directions in general position (i.e. any three directions from $U$ span the whole 3-space) instead; compare the approach to $3 \mathrm{D}$ reconstruction of atomic arrangements presented in [9]. The following conjecture goes back to a continuous version formulated by Gardner in 1995 ([10], Problem 2.1).

Conjecture 2. Let $\Lambda \subset \mathbb{R}^{3}$ be a Delone set. Then, the convex subsets of $\Lambda$ can be distinguished by the X-rays in any set of seven $\Lambda$-directions in general position.

Figure 2 immediately yields that the number seven in Conjecture 2 cannot be lowered to six.
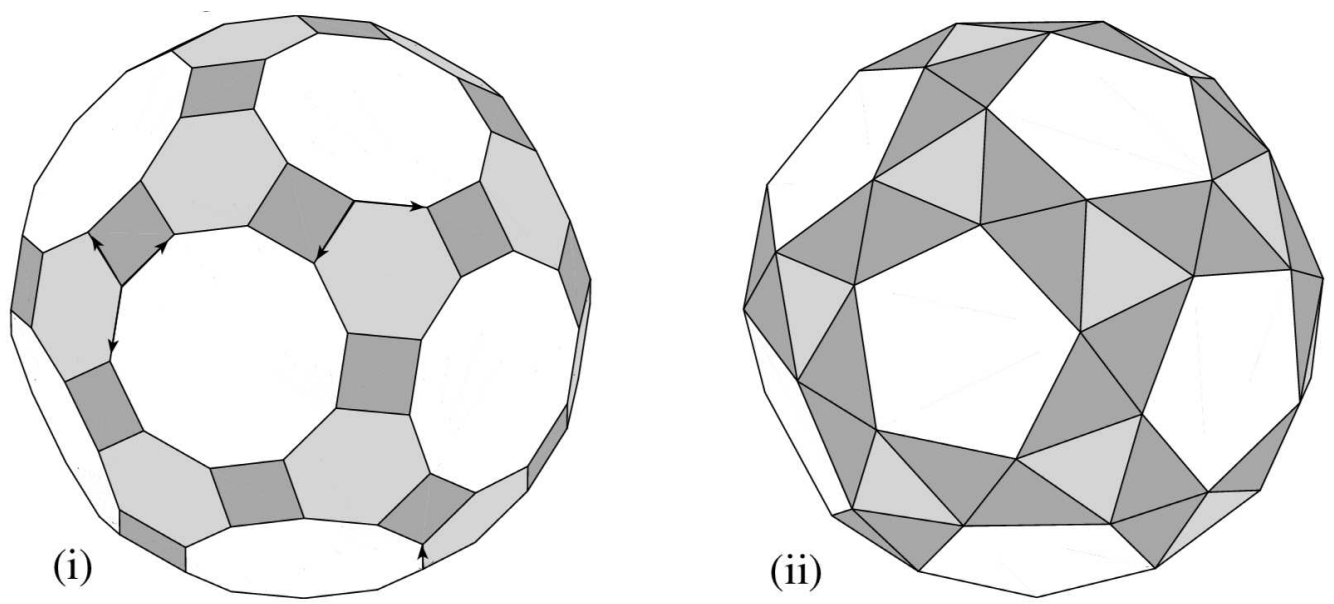

Fig. 2. The great rhombicosidodecahedron (i) (inscribed in the unit sphere) is a $U$-polyhedron (defined just as $U$ polygons) with respect to the indicated set $U$ of six directions in general position. Again, its vertices can be coloured with two colours, say white and grey, such that every white vertex corresponds to a grey vertex, whatever direction from $U$ is used. The convex hull of one colour class is shown in (ii). For symmetry reasons the vertices of (ii) and the vertices of its counterpart (the convex hull of the other colour class) have the same X-rays in the directions of $U$. Figure from [10], p. 66, C Cambridge University Press.

\section{Acknowledgments}

The authors would like to thank Franz Gähler for the help and assistance with the computer calculations. This work was supported by the German Research Foundation (DFG) within the CRC 701.

\section{References}

[1] C. Kisielowski, P. Schwander, F.H. Baumann, M. Seibt, Y. Kim, A. Ourmazd, Ultramicroscopy 58, 131 (1995).

[2] P. Schwander, C. Kisielowski, M. Seibt, F.H. Baumann, Y. Kim, A. Ourmazd, Phys. Rev. Lett. 71, 4150 (1993)

[3] C. Huck, M. Spieß, J. Reine Angew. Math. (Crelle) 677, 199 (2013).
[4] Huck, C. in: Aperiodic Crystals, Eds.: S. Schmid, R.L. Withers, R. Lifshitz, Springer, Dordrecht 2013 , p. 29.

[5] R.J. Gardner, P. Gritzmann, Trans. Am. Math. Soc. 349, 2271 (1997).

[6] M. Baake, U. Grimm, Aperiodic Order. Vol. 1. A Mathematical Invitation, Cambridge University Press, Cambridge 2013.

[7] M. Baake, C. Huck, Philos. Mag. 87, 2839 (2007).

[8] C. Huck, Acta Crystallogr. A 65, 240 (2009).

[9] Y. Ishibashi, H. Sugiura, K. Saitoh, N. Tanaka, Philos. Mag. 91, 2519 (2011).

[10] R.J. Gardner, Geometric Tomography, 2nd ed., Cambridge University Press, New York 2006. 